\title{
Predicting response to oxygen therapy in obstructive sleep apnoea patients using a 10-minute daytime test
}

\author{
David Wang ${ }^{1,2}$, Keith K. Wong ${ }^{1,2}$, Luke Rowsell ${ }^{1}$, Garrick W. Don ${ }^{3}$, \\ Brendon J. Yee ${ }^{1,2}$ and Ronald R. Grunstein ${ }^{1,2}$ \\ Affiliations: ${ }^{1}$ Centre for Integrated Research and Understanding of Sleep (CIRUS), Woolcock Institute of \\ Medical Research, Sydney Medical School, The University of Sydney, Sydney, Australia. ${ }^{2}$ Dept of Respiratory \\ and Sleep Medicine, Royal Prince Alfred Hospital, Sydney Local Health District, Sydney, Australia. ${ }^{3}$ Dept of \\ Respiratory and Sleep Medicine, Royal North Shore Hospita, Sydney, Australia.
}

Correspondence: David Wang, Dept of Respiratory and Sleep Medicine, Royal Prince Alfred Hospital, Missenden Rd, Camperdown, NSW 2050, Australia. E-mail: david.wangdasydney.edu.au

@ERSpublications

A 10-min daytime test may predict the efficacy of $\mathrm{O}_{2}$ in improving the severity of obstructive sleep apnoea http://ow.ly/KZfY30gUXOj

Cite this article as: Wang D, Wong KK, Rowsell L, et al. Predicting response to oxygen therapy in obstructive sleep apnoea patients using a 10-minute daytime test. Eur Respir J 2018; 51: 1701587 [https:// doi.org/10.1183/13993003.01587-2017].

ABSTRACT There is no satisfactory treatment for obstructive sleep apnoea (OSA). Supplemental lowflow oxygen therapy $\left(\mathrm{LFO}_{2}\right)$ has been shown to reduce hypoxaemia and is well tolerated by patients with OSA. However, oxygen therapy may be beneficial only to certain subsets of patients with OSA. In this study, we evaluated a 10-min awake ventilatory chemoreflex test in predicting individual OSA response to 2 months of $\mathrm{LFO}_{2}$ therapy.

At baseline, patients with OSA underwent ventilatory chemoreflex testing in the afternoon, prior to the overnight polysomnography. Subjects were reassessed with polysomnography after 2 months of nocturnal oxygen treatment.

20 patients with OSA completed the study. After 2 months of $\mathrm{O}_{2}$ treatment, changes in the apnoeahypopnoea index (AHI) were significantly correlated with baseline $\mathrm{CO}_{2}$ ventilatory response threshold (VRT) and chemosensitivity $(\mathrm{p}<0.05)$. In predicting a fall in AHI, the area under the receiver operating characteristic curve (AUC) was 0.79 for VRT and 0.89 for chemosensitivity. When these two variables were combined in a logistic regression model, the prediction effect became stronger with an AUC of 0.97, sensitivity of 0.92 and specificity of 0.83 .

Our awake ventilatory chemoreflex test could be considered a simple potential clinical tool to predict individual OSA response to oxygen therapy. It could provide a novel personalised medicine approach to OSA treatment.

Received: Aug 032017 | Accepted after revision: Oct 072017

Support statement: D. Wang was supported by the NHMRC Project Grant (\#1043633); L. Rowsell was supported by the NHMRC CRE NeuroSleep Scholarship (\#1060992); R.R. Grunstein was supported by the NHMRC Senior Principal Research Fellowship. Funding information for this article has been deposited with the Crossref Funder Registry.

Conflict of interest: None declared.

This study is registered as ANZCTR No: 12612000006842.

Copyright @ERS 2018 


\section{Introduction}

Obstructive sleep apnoea (OSA) is a very common disorder affecting up to 50\% of middle-aged men and $23 \%$ of middle-aged women [1]. Untreated OSA causes daytime sleepiness and systemic hypertension, and has been linked to myocardial infarction, congestive heart failure, stroke, diabetes and death [2]. Each year, OSA leads to 1400 road fatalities and a cost of USD15.9 billion in the USA alone [3]. There is no satisfactory treatment for OSA; existing treatment options are 'either poorly tolerated or only partially alleviate abnormalities' [2]. Continuous positive airway pressure (CPAP) is considered the treatment of choice, but its acceptance as a long-term therapy is low, as $46-83 \%$ of patients with OSA are reportedly non-adherent [4]. Although there are a number of alternatives to CPAP therapy, all have their limitations. A major challenge in the field of OSA research is therefore the discovery of acceptable and effective treatments for patients with OSA who reject CPAP.

The pathogenesis of OSA involves a complex interplay between disordered breathing regulation and upper airway obstruction, resulting in intermittent hypoxaemia and sleep fragmentation [5]. A potential alternative to CPAP that has been shown to reduce hypoxaemia, and is relatively well tolerated by patients with OSA, is supplemental low-flow oxygen $\left(\mathrm{LFO}_{2}\right)$ therapy $[6,7]$. Although $\mathrm{LFO}_{2}$ therapy is sometimes used in clinical practice as a salvage therapy in patients with OSA who refuse CPAP [7], its use is limited, because there is no conclusive evidence of an overall beneficial effect on OSA severity and clinical outcomes, despite improvement in oxygenation [7, 8]. The American Academy of Sleep Medicine Standard of Practice Task Force concluded that "supplemental oxygen may benefit subsets of OSA patients" and that one future direction for research is to "characterize the effectiveness of supplemental oxygen in persons with OSA" [9]. Furthermore, given the complex phenotypes of OSA, a recent state of the art review calls for a personalised medicine approach to OSA treatment, with therapy being targeted to more well-defined individual underlying mechanisms [10]. In this context, the ability to predict which patients would benefit from $\mathrm{LFO}_{2}$ therapy would provide a persuasive option for first- or second-line therapy for selected patients with OSA, and potentially reduce the health risks associated with OSA $[9,11]$.

Two small experimental studies from a Harvard group have shown that with one night of oxygen, the severity of sleep apnoea is reduced in patients with OSA, who have a high loop gain measured during sleep $[11,12]$. A further study from the same group showed that overnight upper airway physiological traits could be used to predict OSA response to non-CPAP therapy [13]. A single intervention for one trait could be predicted to treat OSA in approximately one quarter of affected patients and two combined therapies were predicted to treat $\sim 50 \%$ of affected patients [13]. While this study shows a promising research direction, the elegant overnight techniques cannot be easily applied to clinical practice. Ideally, a brief daytime test with a relatively high predictive value would be more useful for clinicians. Previously, we demonstrated that a 10 -min ventilatory response/chemoreflex test while awake could phenotype interindividual OSA response to temazepam [14]. We then further hypothesised that those hypoxia response parameters/ventilatory chemoreflexes should be better predictors of OSA response to supplemental oxygen therapy than temazepam, and therefore conducted the present study. To be more clinically relevant, we tested the OSA responses over a longer term: after 2 months of $\mathrm{O}_{2}$ therapy.

\section{Methods \\ Study protocol and experimental design}

We initially conducted a randomised parallel group double-blind placebo-controlled clinical trial, comparing the effects of supplemental $\mathrm{O}_{2}$ with placebo air in patients with OSA, who refused CPAP. The study was conducted at Royal Prince Alfred Hospital Sleep Laboratory between 2012 and 2014 (ANZCTR No: 12612000006842, SLHD Ethics Committee Approval No: X11-0325\&HREC/11/RPAH/505). At baseline, all included subjects were tested while awake for ventilatory chemoreflex and overnight polysomnography (PSG). The subjects were then blindly assigned to either $\mathrm{O}_{2}$ or air treatments with specially modified, identically appearing, take-home gas concentrators (Air Liquide, Melbourne, Australia). Each night, subjects received $\mathrm{O}_{2}$ /air treatment at a flow rate of $3 \mathrm{~L} \cdot \mathrm{min}^{-1}$ via nasal cannula. 1 week into the intervention phase, arterial blood gas $(\mathrm{ABG})$ was checked for potential $\mathrm{CO}_{2}$ retention. After 2 months of treatment, subjects underwent PSG a second time. After 19 subjects ( 11 on $\mathrm{O}_{2}$, eight on air) were tested, we performed an interim analysis. No overall difference was found in the apnoea-hypopnoea index (AHI) (primary outcome variable) between the $\mathrm{O}_{2}$ and placebo air groups. We found a relationship between baseline awake ventilatory chemoreflexes and the change of $\mathrm{AHI}$ in the $\mathrm{O}_{2}$ group, but not in the air group, suggesting phenotyping potential. We thus changed our research question to confirm the predictive value of the brief daytime test in the selection of OSA responders to $\mathrm{O}_{2}$ therapy. Since this phenotyping effect only occurred with real $\mathrm{O}_{2}$ treatment, we suspended recruitment for the randomised trial, and recruited an additional nine patients with OSA, administering $\mathrm{O}_{2}$ only (uncontrolled and open-label). In total, 20 patients with OSA were treated with 2 months of supplemental $\mathrm{O}_{2}$, and evaluated with ventilatory chemoreflex testing at baseline and PSGs both pre- and post- $\mathrm{O}_{2}$ intervention. 


\section{Participants}

We included OSA subjects between 18-75 years of age, with a total AHI greater than 15 events per h. Subjects either refused or had been previously trialled and failed or were intolerant of CPAP therapy. We excluded patients: who had been using alternative therapy for sleep apnoea, e.g. mandibular advancement splint; with daytime hypoxaemia $\left(\mathrm{SpO}_{2}<95 \%\right)$; with $\mathrm{BMI}>38 \mathrm{~kg} \cdot \mathrm{m}^{-2}$; with severe chronic obstructive pulmonary disease or other respiratory disease; with uncontrolled cardiac disease; who were current smokers; and with severe nasal obstruction.

\section{Awake ventilatory chemoreflex test}

Awake ventilatory chemoreflexes were assessed using our custom-built fully computerised system, based on the Duffin's rebreathing method $[15,16]$. This is a non-invasive breathing test, which includes a 10-min testing session of the iso-oxic hyperoxic ventilatory response to hypercapnia, and a 10-min testing session of the iso-oxic hypoxic ventilatory response to $\mathrm{CO}_{2}$, with a brief resting interval in between the two sessions. The second 10-min test was more important for the present study in terms of $\mathrm{O}_{2}$ effect, and therefore relevant parameters were adopted for final statistical analysis. This test examines ventilatory response to $\mathrm{CO}_{2}$ while holding $\mathrm{PO}_{2}$ constant at $50 \mathrm{mmHg}$ (hypoxia). Each test was performed at a similar time of day and patients were asked to fast for $3 \mathrm{~h}$ prior to the test. The protocol started with $5 \mathrm{~min}$ of hyperventilation with end-tidal $\mathrm{PCO}_{2}$ controlled between $19-25 \mathrm{mmHg}$. If the $\mathrm{PCO}_{2}$ value was $>25 \mathrm{mmHg}$, a red flag would appear on the screen to prompt a faster and deeper breath. If $\mathrm{PCO}_{2}$ was between 19-25 mmHg, a green flag would appear, suggesting an optimal status and indicating that the target range was achieved. If $\mathrm{PCO}_{2}$ was $<19 \mathrm{mmHg}$, a purple flag would appear to prompt slower breathing. After the 5-min hyperventilation period, the computer switched the valve and the subject started rebreathing through a closed circuit containing a gas mixture of $6 \% \mathrm{CO}_{2}$, with $\mathrm{N}_{2}$ making up the balance. During the rebreathing, $\mathrm{PO}_{2}$ in the circuit was held constant at $50 \mathrm{mmHg}$. The computer continuously analysed $\mathrm{O}_{2}$ consumption in the preceding three breaths and used a prediction model to determine how much $\mathrm{O}_{2}$ to feed into the circuit. The rebreathing continued for $5 \mathrm{~min}$ or less, if adequate data points were obtained for subsequent analyses. The $\mathrm{CO}_{2}$ ventilatory response threshold (VRT) and chemosensitivity (the slope of the $\mathrm{PCO}_{2}$ plot against minute ventilation) were analysed through purpose-built software provided by J. Duffin.

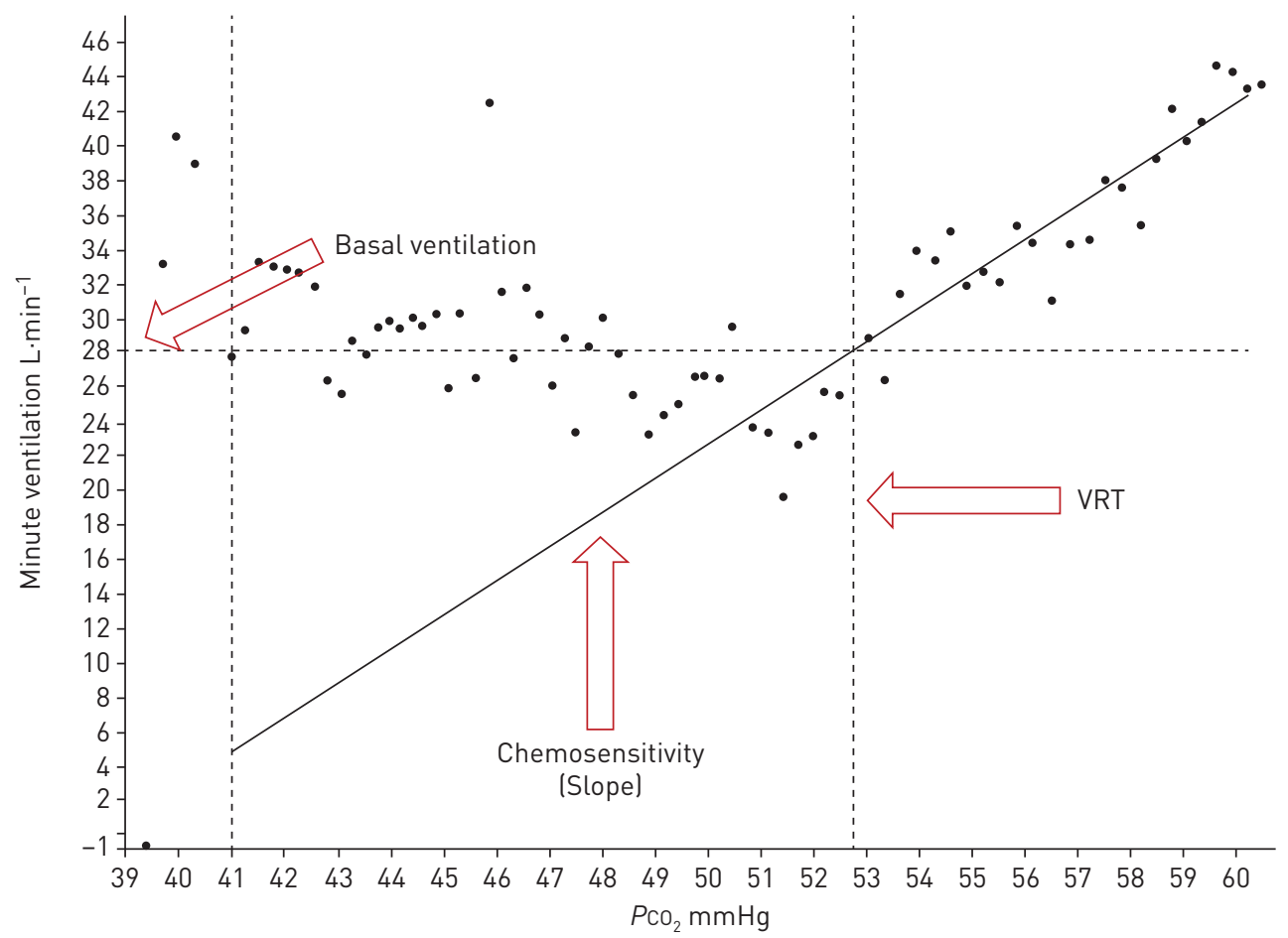

FIGURE 1 Example of a baseline ventilatory chemoreflex test and calculation of the key parameters. The chemosensitivity, ventilatory response threshold (VRT) and basal ventilation were calculated from the protocol of the iso-oxic hypoxic ventilatory response to $\mathrm{CO}_{2}$ [13]. The $\mathrm{PO}_{2}$ in the rebreathing circuit was held constant at $50 \mathrm{mmHg}$ during the test. After $5 \mathrm{~min}$ hyperventilation, subject started to rebreathe in the closed circuit. Minute ventilation did not increase until $P_{\mathrm{CO}_{2}}$ reached response threshold (VRT), and then increased linearly with $\mathrm{PCO}_{2}$. The slope was calculated as the chemosensitivity. The value of minute ventilation at the time of VRT was defined as the basal ventilation. Details of the technique have been described elsewhere [13]. 
An example of the data analysis and definitions of key parameters are presented in figure 1. More detailed procedures have been described in previous publications [14, 17, 18]. The ventilatory chemoreflex data from all patients were independently analysed by D. Wang and L. Rowsell, and results were cross-checked for consistency.

\section{Polysomnogram}

Standard in-lab full PSG were recorded using the Alice 5/G3 Diagnostic Sleep System (Respironics/Philips, Andover, MA, USA). Measurements included six channels of electroencephalogram (EEG), two channels of electro-oculogram (EOG), chin electromyogram (EMG), anterior tibial EMG, electrocardiogram (ECG), body position, nasal pressure, chest and abdomen movements, and arterial oxygen saturation measured by pulse oximetry $\left(\mathrm{S}_{\mathrm{PO}_{2}}\right)$. The PSG results were blindly analysed (with a blind code that anonymised the names of the patients and order of treatment) by an experienced sleep scientist, using both standard American Academy of Sleep Medicine (AASM) criteria [19] and a modified criteria that ignored 3\% of $\mathrm{SpO}_{2}$ desaturation as a criterion when scoring hypopnoea. Thus, the hypopnoea criteria for the standard AHI required a peak respiratory flow drop $\geqslant 30 \%$, and either a $\geqslant 3 \%$ oxygen desaturation or an arousal [19], whereas a hypopnoea event for the modified AHI must have been associated with an arousal (desaturation only hypopnoeas were ignored).

\section{Statistical analysis}

Our primary outcome variable was AHI [19]. Pre- and post- $\mathrm{O}_{2}$ treatment PSG respiratory variables were compared using the paired t-test. We correlated baseline ventilatory chemoreflex parameters (VRT and chemosensitivity) with the change in AHI after 2 months of $\mathrm{O}_{2}$ therapy, and calculated standard diagnostic test statistics (sensitivity, specificity, area under the receiver operating characteristic curve (AUC)) with respect to response to $\mathrm{O}_{2}$ therapy (defined as a decline in AHI). Pearson's correlation coefficient was used to test preliminary correlations. We also explored logistic regression models combining individual variables from ventilatory response testing (basal ventilation, ventilatory response threshold, and chemosensitivity; figure 1) in the prediction of response to $\mathrm{O}_{2}$ therapy. The likelihood ratio Chi-squared test was used to find significant predictors. Statistical analysis was conducted using the SPSS 19 and R-Project (www.R-project.org) software. Data were presented as mean \pm SD unless otherwise stated. The values of $\mathrm{p}<0.05$ were considered statistically significant.

\section{Results}

The 20 subjects ( 14 males, six females) were $57.8 \pm 10.9$ years of age, with a BMI of $31 \pm 9$ (range 2461) $\mathrm{kg} \cdot \mathrm{m}^{-2}$ and baseline AHI of $32.6 \pm 21.9(5-107) \mathrm{h}^{-1}$. Their baseline and post $-\mathrm{O}_{2}$ treatment PSG parameters are shown in table 1. For baseline ventilatory chemoreflex tests (iso-oxia hypoxia protocol), patients had an average chemosensitivity (slope) of $3.46 \pm 2.04 \mathrm{~L} \cdot \mathrm{min}^{-1} \cdot \mathrm{mmHg}^{-1}$, VRT of 48.15 $\pm 4.13 \mathrm{mmHg}$, and basal ventilation of $16.39 \pm 5.65 \mathrm{~L} \cdot \mathrm{min}^{-1}$.

As primary outcome of interest, change in AHI was significantly correlated with baseline VRT $(\mathrm{r}=-0.48$, $\mathrm{p}=0.04)$, chemosensitivity $(\mathrm{r}=0.50, \mathrm{p}=0.03)$, and basal ventilation $(\mathrm{r}=0.50 \mathrm{p}=0.03)$ after 2 months of $\mathrm{O}_{2}$ treatment in patients with OSA (table 2, figure 2). In predicting a decline in $\mathrm{AHI}$ after $\mathrm{O}_{2}$ therapy (change in $\mathrm{AHI}<0$ ), the AUC was 0.79 for VRT and 0.89 for chemosensitivity. Importantly, when these two variables were combined in a logistic regression model, the prediction effect became stronger with a sensitivity of 0.92 and specificity of 0.83 (table 3). Basal ventilation was not a significant predictor in the

TABLE 1 Comparison of polysomnography respiratory parameters before and after 2 months of oxygen treatment

\begin{tabular}{lccc} 
& Baseline & Post $\mathbf{0}_{\mathbf{2}}$ treatment & p-value \\
\hline AHI per $\mathbf{h}$ & $32.6 \pm 21.9$ & $24.2 \pm 22.2$ & 0.068 \\
AHI (modified criteria) per $\mathbf{h}$ & $30.7 \pm 22.2$ & $23.0 \pm 22.0$ & 0.095 \\
Average $\mathrm{SpO}_{\mathbf{2}} \%$ & $93.6 \pm 1.4$ & $96.7 \pm 2.1$ & $<0.001$ \\
ODI per $\mathbf{h}$ & $25.3 \pm 18.0$ & $11.6 \pm 25.2$ & 0.014 \\
T90 min & $10.0 \pm 12.1$ & $4.3 \pm 10.8$ & 0.134 \\
Arousal index per $\mathbf{h}$ & $27.5 \pm 13.7$ & $33.0 \pm 22.6$ & 0.275
\end{tabular}

Data are presented as mean \pm SD. AHI: apnoea-hypopnoea index (modified criteria ignored $3 \%$ of desaturation as a criterion to mark hypopnoea); $\mathrm{SpO}_{2}$ : arterial oxygen saturation measured by pulse oximetry; ODI: oxygen desaturation index; T90: sleep time with $\mathrm{SpO}_{2}<90 \%$. p-value was calculated by the paired t-test. 
TABLE 2 Correlations between key baseline ventilatory chemoreflex parameters and change in obstructive sleep apnoea severity after 2 months of $\mathrm{O}_{2}$ treatment

\section{Change of $\mathrm{AHI}$}

\begin{tabular}{|c|c|c|c|c|}
\hline & \multirow[b]{2}{*}{$r$} & \multirow[b]{2}{*}{ p-value } & \multicolumn{2}{|c|}{ criteria) } \\
\hline & & & $\mathbf{r}$ & p-value \\
\hline Chemosensitivity L. $\min ^{-1} \cdot \mathrm{mmHg}^{-1}$ & 0.503 & 0.033 & 0.498 & 0.042 \\
\hline VRT $\mathrm{mmHg}$ & -0.483 & 0.043 & -0.404 & 0.107 \\
\hline Basal ventilation L.min ${ }^{-1}$ & 0.501 & 0.034 & 0.518 & 0.033 \\
\hline
\end{tabular}

AHI: apnoea-hypopnoea index Imodified criteria ignored $3 \%$ of desaturation as a criterion to mark hypopnoea); VRT: $\mathrm{CO}_{2}$ ventilatory response threshold. Pearson's correlation coefficient was used to test correlations. Significant $\mathrm{p}$-values are present in bold type.

multivariate model $(\mathrm{p}=0.31)$. Figure 3 shows the receiver operating characteristic curve for the two variables combined (AUC=0.97, 95\% CI 0.91-1.0). Based on the data from 68 patients in three of our OSA studies (current study and previous studies [14, 17]) who underwent ventilatory response testing at baseline, 25\% of patients with OSA had a pattern of chemosensitivity and VRT that would be expected to respond to oxygen, defined as an improvement in AHI.
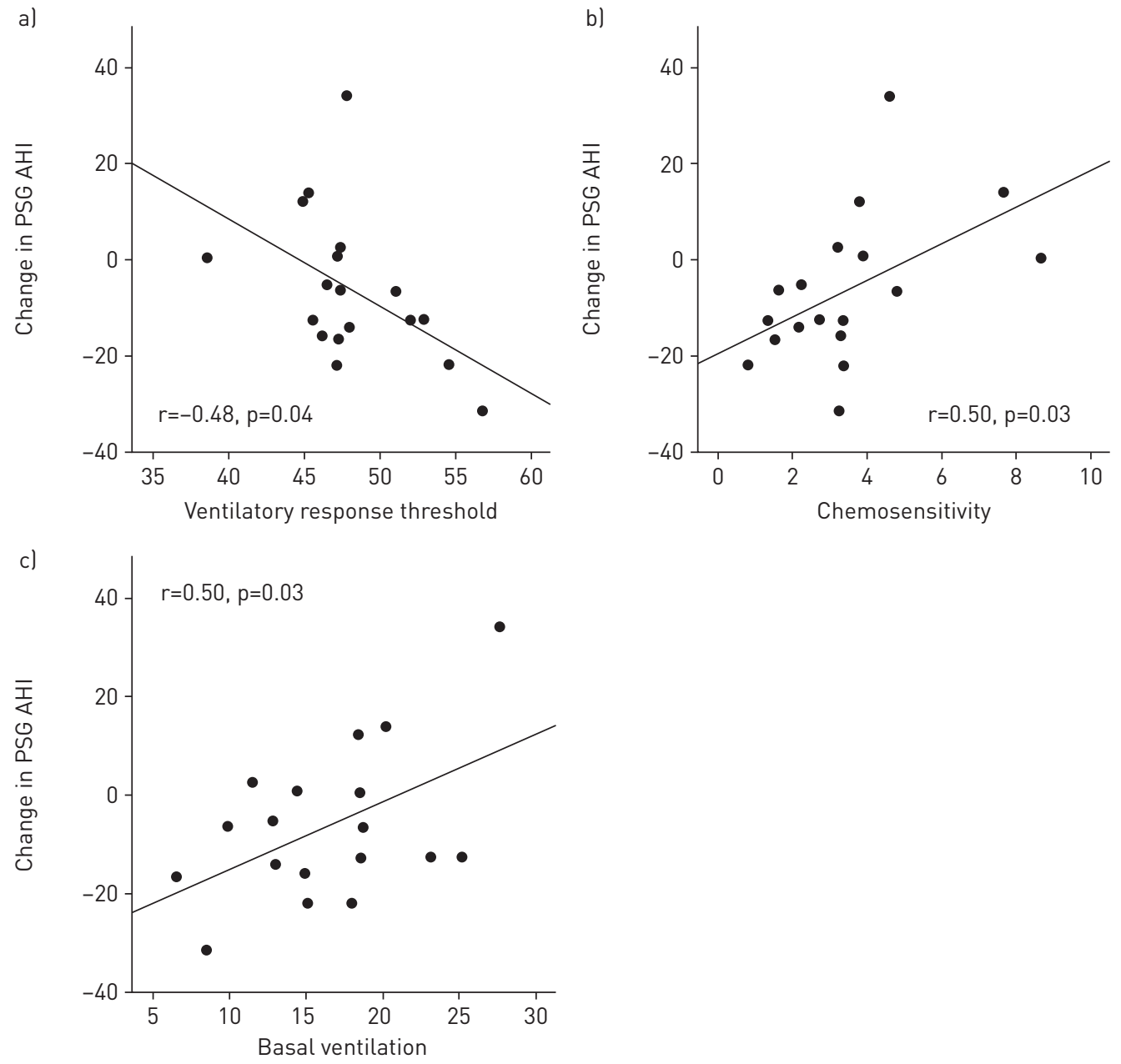

FIGURE 2 Significant associations between three key baseline ventilatory chemoreflex parameters and changes in the apnoea-hypopnoea index (AHI) during sleep in obstructive sleep apnoea (OSA) patients after receiving 2 months $\mathrm{O}_{2}$ therapy. Change refers to the difference between post-treatment and pre-treatment. PSG: polysomnography. 
TABLE 3 Predictive value of key variables of awake ventilatory chemoreflex test and the two variables combined through a logistic regression model

\begin{tabular}{lcccc} 
& Chemosensitivity & VRT & Two variables combined & Basal ventilation \\
\hline Sensitivity & 0.92 & 0.58 & 0.92 & 0.67 \\
Specificity & 0.83 & 0.83 & 0.83 & 0.67 \\
AUC & 0.89 & 0.79 & 0.97 & 0.64 \\
p-value & 0.0048 & 0.011 & & 0.31
\end{tabular}

VRT: $\mathrm{CO}_{2}$ ventilatory response threshold; $A \cup C$ : area under the receiver operating characteristic curve. p-value was calculated using the likelihood ratio Chi-squared test.

To investigate potential $\mathrm{CO}_{2}$ retention, we also analysed $\mathrm{ABG}$ in the controlled trial phase, 1 week into the intervention. The 11 patients with OSA who received $\mathrm{O}_{2}$ therapy had an average $\mathrm{PCO}_{2}$ of $39.6 \pm 3.8$ (range 35-48) $\mathrm{mmHg}$, whereas the eight patients who received the placebo air treatment had an average $P_{\mathrm{CO}_{2}}$ of $41.9 \pm 2.0$ (range $38-44) \mathrm{mmHg}(\mathrm{p}=0.15)$. Only one patient in the $\mathrm{O}_{2}$ group had an awake $\mathrm{ABG}$ $\mathrm{PCO}_{2}>45 \mathrm{mmHg}(48 \mathrm{mmHg})$.

\section{Discussion}

This study has demonstrated for the first time that a 10 -min non-invasive ventilatory chemoreflex test performed during wakefulness could predict the response to low-flow oxygen therapy in patients with OSA. This finding could provide a clinically applicable personalised medicine approach to aid clinicians in selecting suitable patients for low-flow oxygen therapy, a treatment with potentially better compliance than CPAP.

An obvious question was how a brief 10-min breathing test during the day might predict the number of times an OSA patient would stop breathing during sleep, with oxygen use. During sleep, breathing is largely controlled by neurochemical chemoreceptor feedback $[5,20]$. Assessment of awake ventilatory chemoreflexes provides important information on the feedback of central and peripheral chemoreceptors, the control system responsible for $\mathrm{PaCO}_{2}$ and $\mathrm{PaO}_{2}$ homeostasis. Of the two fundamental factors in OSA pathogenesis, increased airway collapsibility and enhanced ventilatory control system instability, the former is not necessary to generate a cyclical pattern of OSA. This cyclical pattern depends on how a patient's respiratory control system responds to the airway obstruction [5]. Awake ventilatory chemoreflex tests (including our test) are usually designed to assess ventilatory response to hypercapnia and hypoxia [16]. Moreover, breathing control instability during sleep involves two major factors: one is enhanced ventilatory chemosensitivity (controller gain); the other is a narrowing in $\mathrm{CO}_{2}$ reserve, which is the $\mathrm{PCO}_{2}$ difference between the apnoea and eupnoea thresholds [21]. Therefore, an ideal awake chemoreflex test

FIGURE 3 Receiver operating characteristic curve examining the two-variable combined logistic regression model, with respect to oxygen therapy by a polysomnography apnoea-hypopnoea index change of $<0$. The two variables considered were chemosensitivity and ventilatory response threshold. The area under the curve was 0.97 .

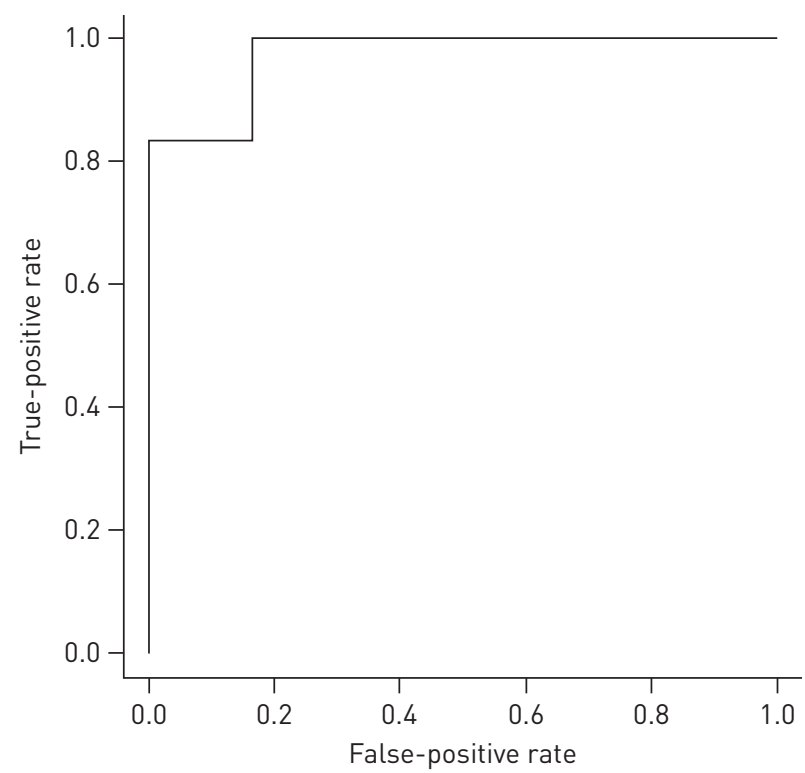


should measure both chemosensitivity and $\mathrm{CO}_{2}$ thresholds. While there are a few versions of ventilatory chemoreflex tests that measure chemosensitivity, Duffin's modified rebreathing method is the only method that can directly measure both parameters $[15,16]$. We believe that the measure of $\mathrm{CO}_{2} \mathrm{VRT}$ is comparable to the concept of eupnoea threshold during sleep, and chemosensitivity is comparable to the concept of controller gain and sub-threshold chemosensitivity during sleep apnoea [18, 22]. Our system was designed and modified based on the Duffin method as described in the Methods section [15]. We developed a fully computerised chemoreflex testing system, where the test procedure is software-standardised and human operating variability is minimised. To maintain a steady control of iso-hypoxia, we developed an $\mathrm{O}_{2}$ consumption-prediction model in the operating software, thereby allowing continuous prediction of the consumption of $\mathrm{O}_{2}$ in the fourth breath, based on the previous three breaths [14]. This automated the control of $\mathrm{O}_{2}$ entry into the testing system, thereby avoiding problems with $\mathrm{PO}_{2}$ fluctuation caused by the manual feeding of $\mathrm{O}_{2}$. These innovations allowed us to closely simulate overnight breathing control physiology in patients with OSA using a brief daytime test.

With this system, we demonstrated close links between awake ventilatory chemoreflexes and the changes observed in sleep disordered breathing in a few studies $[14,17,18,23,24]$. Under a double-blind, placebocontrolled cross-over design, we found that baseline central chemosensitivity measurement could phenotype patients with OSA who are prone to greater respiratory depression after taking $10 \mathrm{mg}$ temazepam [14]. In another study that tested the effect of a single mild dose of morphine on OSA, we showed that awake VRT was significantly correlated with plasma morphine concentrations and time spent with $\mathrm{SpO}_{2}<90 \%$ (T90) during sleep [18]. In another study that tested the effect of testosterone on OSA, we found significant correlations between change in VRT and T90 during sleep, and between VRT and serum testosterone [17]. In a more recent double-blind placebo-controlled cross-over study that investigated the effect of morphine on OSA, we found that in patients with severe OSA, lower baseline VRT was significantly correlated with worsening sleep time with $\mathrm{SpO}_{2}<90 \%$, AHI, oxygen desaturation index and arousal index with $40 \mathrm{mg}$ of slow-release oral morphine [24]. This suggests that our awake ventilatory chemoreflex test also has the potential to predict which patients with OSA are prone to respiratory failure and arrest during sleep with opioid use. We believe these phenotyping/predicting effects should be stronger in testing the effect of oxygen on OSA, as our ventilatory chemoreflex test directly evaluates the responses to hypoxia and hypercapnia.

Our study is designed to test OSA response after 2 months of low-flow $\mathrm{O}_{2}$ therapy, and the long-term treatment of the intervention is close to a real world scenario. As shown in figure 2, patients with higher baseline chemosensitivity had greater OSA severity with oxygen. This finding is not consistent with other experimental studies that tested single-night $\mathrm{O}_{2}$ effects on OSA, using the loop gain test measured during sleep. Wellman et al. [12] compared the effect of single-night $\mathrm{O}_{2}$ on six patients with OSA with high loop gain, and six patients with low loop gain. Oxygen reduced loop gain, and lowered AHI in the high loop gain group, and had no effect on the low loop gain group [12]. Another study from the same group [11] evaluated the effect of single-night $\mathrm{O}_{2}$ and hypoxia on loop gain in 11 patients with OSA. They found that $\mathrm{O}_{2}$ lowered loop gain because of a reduction in controller gain, and had no effect on plant gain. Furthermore, $\mathrm{O}_{2}$ did not affect other key physiological traits, such as upper airway responsiveness, pharyngeal collapsibility and arousal threshold. They speculated that the beneficial effect of $\mathrm{O}_{2}$ on the severity of OSA is primarily based on its ability to reduce loop gain [11]. While our awake chemosensitivity is comparable to the concept of controller gain/loop gain, these studies showed that for phenotyping OSA response to $\mathrm{O}_{2}$, it is more feasible to focus on ventilatory chemosensitivity/loop gain/ controller gain, rather than other physiological traits. Moreover, it is difficult to compare the single-night effects of oxygen with the effects of 2 months of oxygen therapy. Human responses to acute and long-term oxygen can be very different, if not opposite: a typical example is seen in first-night sleep at high altitude, during which hypoxia may cause augmented hypoxic ventilatory response/chemosensitivity/controller gain, which is a major mechanism for high altitude central sleep apnoea; conversely, long-term exposure to high altitude hypoxia may result in blunted hypoxic responses [20]. It is certainly desirable to test single-night effects of $\mathrm{O}_{2}$, using our protocol or test loop gain after 2 months of $\mathrm{O}_{2}$ treatment, to better understand the time course of the underlying mechanism. Under a retrospective design, a later study from the Wellman group [13] analysed 57 subjects either with or without OSA, who had been evaluated for overnight upper airway physiological traits under different non-CPAP interventions. The traits were incorporated into a physiological model, which had a good sensitivity (80\%) and specificity (100\%) for predicting OSA. A single intervention on one trait could have been predicted to treat OSA in around $25 \%$ of the patients with OSA. The reported performance of this model is very similar to that of our study. While both studies demonstrated the potential use of baseline physiological measures to predict OSA response to interventions, our ventilatory chemoreflex test, as a quick and non-invasive test during wakefulness, would be much easier to perform in clinical settings. 
A major side effect of oxygen therapy is potential $\mathrm{CO}_{2}$ retention. An early study showed that one month of supplemental oxygen $\left(4 \mathrm{~L} \cdot \mathrm{min}^{-1}\right.$ ) on average, increased waking $\mathrm{PaCO}_{2}$ by $3 \mathrm{mmHg}$ compared to placebo air treatment in eight patients with OSA [25]. In our study, only one out of 11 patients had a $\mathrm{PaCO}_{2}$ higher than $45 \mathrm{mmHg}$, one week after $\mathrm{O}_{2}$ treatment $(48 \mathrm{mmHg})$. This is similar to the finding from a recent controlled trial showing that $11.4 \%$ of 62 post-operative patients with OSA who received $3 \mathrm{~L} \cdot \mathrm{min}^{-1} \mathrm{O}_{2}$ had increased overnight transcutaneous $\mathrm{CO}_{2}\left(\mathrm{PtcCO}_{\mathrm{tc}}\right)$. However, no group differences were observed in $\mathrm{PtcCO} 2$ compared to 61 control patients with OSA, who had not received an oxygen intervention [26]. Nevertheless, our mildly increased $\mathrm{PCO}_{2}$ might have contributed to the slightly increased arousal index, despite the mild decrease in AHI, as seen in table 1. Arousal during sleep is known to be easily triggered by hypercapnia, but not hypoxia [27].

The present study is limited by the small sample size and a proof-of-concept study design. A larger sample size with a broad spectrum of OSA severity would allow us to assess the prediction value of different cut-off points in AHI, such as a larger decline in AHI, which could be more clinically meaningful. Further studies that are more comprehensive are recommended to validate our predictive power and extend the test utility to other clinical outcomes of OSA, i.e. neurocognitive and cardiovascular outcomes, particularly to explore the clinical outcome response to the mildly increased $\mathrm{CO}_{2}$ from the low-flow oxygen therapy. The data obtained could inform a large scale randomised controlled trial to establish cost-benefit. This next phase could also include the development of simplified and miniaturised chemoreflex testing devices capable of measuring key respiratory control parameters in an office setting.

In conclusion, there could be an intrinsic physiological link between our 10-min daytime test and breathing during sleep in patients with OSA. Our awake ventilatory chemoreflex test could potentially become a clinically applicable tool to predict individual OSA response to oxygen therapy, with high sensitivity and specificity. It could provide a novel personalised medicine approach for selection of suitable patients with OSA for targeted treatment, especially those patients with OSA who reject CPAP therapy.

\section{Acknowledgements}

We would like to thank the staff of the Sleep Laboratory, Royal Prince Alfred Hospital who assisted in completing the study. Take-home $\mathrm{O}_{2}$ and placebo air gas concentrators were provided without charge by Air Liquide, Australia.

D. Wang, K.K. Wong, B.J. Yee and R.R. Grunstein contributed to the design of the study. The manuscript was written by D. Wang, and all authors read, revised and approved the final version. Statistical analyses were performed by K.K. Wong. Recruitment and data collection was conducted by G.W. Don, L. Rowsell and D. Wang. Ventilatory chemoreflex testing and analysis was supervised by D. Wang. All authors approved the final version of the manuscript, and agreed to be accountable for all aspects of the work in ensuring that questions related to the accuracy or integrity of any part of the work were appropriately investigated and resolved. All persons designated as authors qualify for authorship, and all those who qualify for authorship are listed.

\section{References}

1 Heinzer R, Vat S, Marques-Vidal P, et al. Prevalence of sleep-disordered breathing in the general population: the HypnoLaus study. Lancet Respir Med 2015; 3: 310-318.

2 Jordan AS, McSharry DG, Malhotra A. Adult obstructive sleep apnoea. Lancet 2014; 383: 736-747.

3 Sassani A, Findley LJ, Kryger M, et al. Reducing motor-vehicle collisions, costs, and fatalities by treating obstructive sleep apnea syndrome. Sleep 2004; 27: 453-458.

4 Weaver TE, Grunstein RR. Adherence to continuous positive airway pressure therapy: the challenge to effective treatment. Proc Am Thorac Soc 2008; 5: 173-178.

5 Dempsey JA, Xie A, Patz DS, et al. Physiology in medicine: obstructive sleep apnea pathogenesis and treatment-considerations beyond airway anatomy. J Appl Physiol (1985) 2014; 116: 3-12.

6 Loredo JS, Ancoli-Israel S, Kim EJ, et al. Effect of continuous positive airway pressure versus supplemental oxygen on sleep quality in obstructive sleep apnea: a placebo-CPAP-controlled study. Sleep 2006; 29: 564-571.

7 Gottlieb DJ, Punjabi NM, Mehra R, et al. CPAP versus oxygen in obstructive sleep apnea. N Engl J Med 2014; 370: 2276-2285.

8 Mehta V, Vasu TS, Phillips B, et al. Obstructive sleep apnea and oxygen therapy: a systematic review of the literature and meta-analysis. J Clin Sleep Med 2013; 9: 271-279.

9 Veasey SC, Guilleminault C, Strohl KP, et al. Medical therapy for obstructive sleep apnea: a review by the Medical Therapy for Obstructive Sleep Apnea Task Force of the Standards of Practice Committee of the American Academy of Sleep Medicine. Sleep 2006; 29: 1036-1044.

10 Javaheri S, Barbe F, Campos-Rodriguez F, et al. Sleep apnea: types, mechanisms, and clinical cardiovascular consequences. J Am Coll Cardiol 2017; 69: 841-858.

11 Edwards BA, Sands SA, Owens RL, et al. Effects of hyperoxia and hypoxia on the physiological traits responsible for obstructive sleep apnoea. J Physiol 2014; 592: 4523-4535.

12 Wellman A, Malhotra A, Jordan AS, et al. Effect of oxygen in obstructive sleep apnea: role of loop gain. Respir Physiol Neurobiol 2008; 162: 144-151.

13 Owens RL, Edwards BA, Eckert DJ, et al. An integrative model of physiological traits can be used to predict obstructive sleep apnea and response to non positive airway pressure therapy. Sleep 2015; 38: 961-970.

14 Wang D, Marshall NS, Duffin J, et al. Phenotyping interindividual variability in obstructive sleep apnoea response to temazepam using ventilatory chemoreflexes during wakefulness. J Sleep Res 2011; 20: 526-532. 
Duffin J, Mohan RM, Vasiliou P, et al. A model of the chemoreflex control of breathing in humans: model parameters measurement. Respir Physiol 2000; 120: 13-26.

16 Duffin J. Measuring the respiratory chemoreflexes in humans. Respir Physiol Neurobiol 2011; 177: 71-79.

17 Killick R, Wang D, Hoyos CM, et al. The effects of testosterone on ventilatory responses in men with obstructive sleep apnea: a randomised, placebo-controlled trial. J Sleep Res 2013; 22: 331-336.

18 Wang D, Somogyi AA, Yee BJ, et al. The effects of a single mild dose of morphine on chemoreflexes and breathing in obstructive sleep apnea. Respir Physiol Neurobiol 2013; 185: 526-532.

19 Berry RB, Budhiraja R, Gottlieb DJ, et al. Rules for scoring respiratory events in sleep: update of the 2007 AASM Manual for the Scoring of Sleep and Associated Events. Deliberations of the Sleep Apnea Definitions Task Force of the American Academy of Sleep Medicine. J Clin Sleep Med 2012; 8: 597-619.

20 Dempsey JA, Veasey SC, Morgan BJ, et al. Pathophysiology of sleep apnea. Physiol Rev 2010; 90: 47-112.

21 Dempsey JA. Crossing the apnoeic threshold: causes and consequences. Exp Physiol 2005; 90: 13-24.

22 Mateika JH, Omran Q, Rowley JA, et al. Treatment with leuprolide acetate decreases the threshold of the ventilatory response to carbon dioxide in healthy males. J Physiol 2004; 561: 637-646.

23 Wang D, Eckert DJ, Grunstein RR. Drug effects on ventilatory control and upper airway physiology related to sleep apnea. Respir Physiol Neurobiol 2013; 188: 257-266.

24 Wang D, Rowsell L, Wong KK, et al. Identifying obstructive sleep apnea patients vulnerable to opioid-induced respiratory depression - a randomized double-blind placebo-controlled crossover trial. Am J Respir Crit Care Med 2016; 193: A4321.

25 Gold AR, Schwartz AR, Bleecker ER, et al. The effect of chronic nocturnal oxygen administration upon sleep apnea. Am Rev Respir Dis 1986; 134: 925-929.

26 Liao P, Wong J, Singh M, et al. Postoperative oxygen therapy in patients with OSA: a randomized controlled trial. Chest 2017; 151: 597-611.

27 Berthon-Jones M, Sullivan CE. Ventilation and arousal responses to hypercapnia in normal sleeping humans. $J$ Appl Physiol Respir Environ Exerc Physiol 1984; 57: 59-67. 